\title{
Fast Algorithm for Multimodal Image Registration with DTCWT
}

\author{
Venkateshappa* and P. Cyril Prasanna Raj \\ Department of Electronics and Communication Engineering, M S Engineering College, Bengaluru - 562110, \\ Karnataka, India; venkat_harishith@rediffmail.com, cyril@msec.ac.in
}

\begin{abstract}
Objective: To perform image registration with sub band features computed using dual tree complex wavelets and reducing the computation time in registration. Methods/Statistical Analysis: The input images are preprocessed with Gaussian filters; features are extracted from dual tree sub bands. The dominant features are retained with feature selection process to minimize complexity in registration. Translation of images based on similar features is carried out using singular value decomposition. A refinement phase further registers the images considering similarities in features. The proposed algorithm is modeled in MATLAB, registration processes are modeled from fundamental equations. Test images are considered for validation. The test images are geometrically transformed with knows sets of translation, scale and rotational parameters. The two images are registered and the corresponding transformation parameters are compared. Critical points in the images are considered as reference points for validation. Findings: The proposed algorithm performs registration of all images with less than $10 \%$ error. The PSNR computed for all sets of data base is greater than $32 \mathrm{~dB}$. With dual tree sub bands, the unique features with $15^{\circ}, 45^{\circ}$ and $75^{\circ}$ orientations are captured thus the features are twice more compared with DWT features. The feature selection algorithm considers the orientations in all three directions thus improving registration process. SVD algorithm optimizes the complexity in transformation process and refinement phase. Elimination of false features and use of appropriate sub band features has reduced the registration time by $76 \%$ thus meeting the requirement for real time applications in micro air vehicles. Application/Improvements: The improvement in computation time and registration accuracy of this algorithm is suitable for MAVs moving with speed of 20-30kmph. Multilevel decomposition will further improve the registration process, hardware accelerators can reduce computation time to few milliseconds.
\end{abstract}

Keywords: Autonomous Navigation, Dual Tree Complex Wavelets, Feature Extraction, Image Registration, SVD

\section{Introduction}

Satellite images are used in applications like astronomy, geosciences studies and geographical information systems. Registration of satellite images helps in information extraction that provides accurate data for autonomous navigation. Image representation of data captured by image sensors provides visual interpretation of information. For time critical applications such as remote sensing, medical imaging and border security, registration algorithm needs hardware accelerators. Image registration consists of four steps: Feature extraction, feature match- ing, transform estimation and resampling transformation. The input image is decomposed into four sub bands using wavelet transform, in order to reduce registration time edge features are selected from the HL and LH band for registration 1 . The selected features from sub bands are further tuned by retaining significant features based on local modulus maxima and thresholding, thus reducing computation time $e^{2}$. Techniques such as maximum correlation coefficients and affine transformation are used for feature matching and transformation to improve image registration 2 . Registration results are further improved with techniques such as bilinear interpolation and mutual

${ }^{*}$ Author for correspondence 
correlation ${ }^{3}$. The wavelet sub bands provide features such as edges at multiple resolutions, in order to select the best features voting algorithm is proposed that selects the edge features based on intensity and normalized correlation ${ }^{4}$ and mutual information metric ${ }^{\frac{5}{}}$ is used for similarity measurement ${ }^{4}$.

Wavelet transforms combined with allied techniques enhances the performance metrics of image registration. However, there are certain limitations in wavelets that are addressed by dual tree wavelet transforms ${ }^{\underline{6}}$. If the image to be registered have shifts as compared with reference images wavelets exhibit shift variance property thus altering the energy coefficients across the wavelet sub bands. Wavelet sub bands have three orientations of $0^{\circ}, 45^{\circ}$ and $90^{\circ}$. Registration of images with directional features and shift invariance will improve registration results and hence use of DTCWT is considered in this work.

DTCWT for image processing has been demonstrated to be superior over that of wavelets in terms of shift insensitive and directionality ${ }^{7.8}$. These properties of dual tree wavelets has advantages in image processing such as denoising, deblurring, super-resolution, watermarking ${ }^{9}$, segmentation ${ }^{10}$ and pattern classification ${ }^{11}$. DWT loses its shift insensitive property due to down sampling, further with separability in decomposition directional selectivity in DWT is lost $\frac{12,13}{13}$. Two Dimensional (2D) DTCWT produces directionally selective sub bands that can be used to capture features in the images for registration. Multimodal images such as IR and visible images have been registered using dual tree wavelets ${ }^{14}$. The images are decomposed into sub bands, from the sub band pyramids feature are extracted based on edge information at the lowest level sub band and is used as matching criteria ${ }^{14}$. The search criteria are refined at higher levels based on mutual information thus reducing the computational time for search operations. Accuracy in registration is improved to $25 \%^{14}$ as compared with wavelet based registration technique. A novel approach for registration based on wavelets performs preprocessing using gradients in both $\mathrm{x}$ and $\mathrm{y}$ directions on the input image. Features are extracted using wavelets from the preprocessed image thus improving performance ${ }^{15}$. The pyramidal approach is best as it uses features from lowest bands in the pyramid for registration and fine tuning is performed with features from bands higher in the pyramid. Preprocessing normalizes intensity distribution in input images. The directional features provide additional features for two step image registration. The additional features can be obtained using dual tree wavelets. In this paper a novel approach is proposed for IR based on DTCWT that produces low pass sub band (real and complex) and six complex sub bands with orientations of $\left( \pm 15^{\circ}, \pm 45^{\circ}\right.$ and $\left.\pm 75^{\circ}\right)$. The features in sub bands are selected optimally for coarse and fine registration.

\section{Design and Methodology}

Input image acquired would have noise, hence Gaussian filtering is performed to smoothen the noise in the image, by performing averaging technique, enhancing the feature selection to select better points in both the input and reference image. In order to filter noise Gaussian filter is selected and is convolved with the input image as in Eq. (1). The filtered image is processed further and is registered as per the proposed algorithm. From the results obtained it was found that the registered image that was preprocessed using Gaussian filter achieved PSNR of 39.8 as compared with registered image without Gaussian filter with PSNR of $35.5 \mathrm{~dB}$. Gaussian filtering filters the noise and normalizes the image assisting feature selection and thus providing feature points for registration. A Gaussian mask of size $(m, n)$ is created, and convolution operation is carried out as in Eq. (1).

$G(x, y) * I(x, y)=\sum_{x=-a}^{a} \sum_{x=-b}^{b} G(s, t) I(x-s, y-t)$

Gaussian constant $\sigma=1$, the Gaussian mask is set to $21 \times 21$, for an image of size N1 x N2 the Gaussian mask is convolved without overlap. The Gaussian mask is selected such that the filtering is optimum and also requires less time to process.

\subsection{DTCWT Computation}

The Gaussian filtered image is decomposed using DTCWT 11, 17 tap symmetric filters. The images are decomposed to three levels, with each level consisting of real part and imaginary part. The imaginary part consists of six orientation bands with $\left( \pm 15^{\circ}, \pm 45^{\circ}\right.$ and $\left.\pm 75^{\circ}\right)$.

The input image $\mathrm{X}$ is decomposed into eight sub bands as shown in Figure 1, the first stage consists of row processing, and the second stage consists of column processing filters. The butterfly structure is designed with sign inversion operation and 2's complement operation to reduce computation complexity. The division operation by $\sqrt{2}$ is replaced with threshold operation. The proposed 
algorithm performs three levels decomposition of input image and reference image as shown in Figure 2.

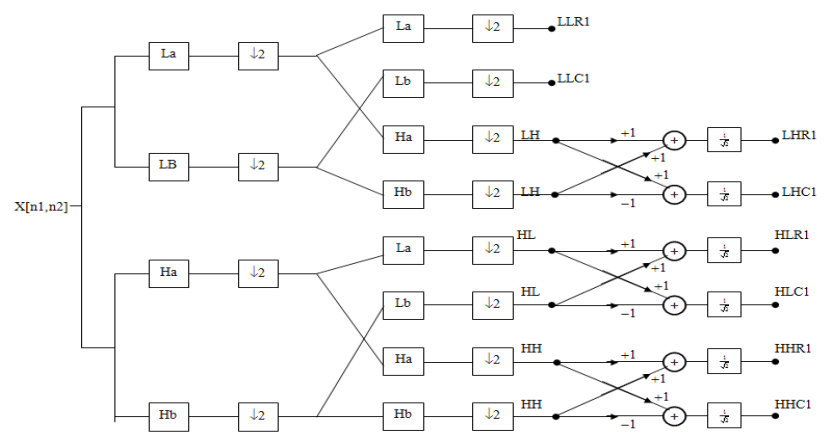

Figure 1. DTCWT computation.

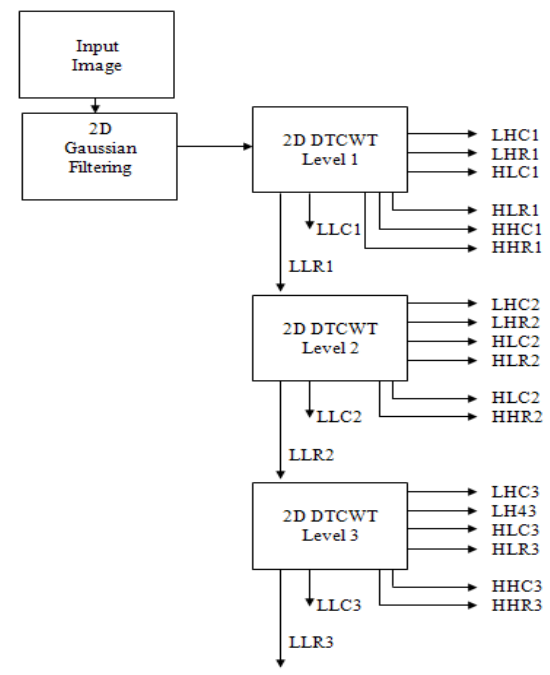

Figure 2. Three level DTCWT sub bands.

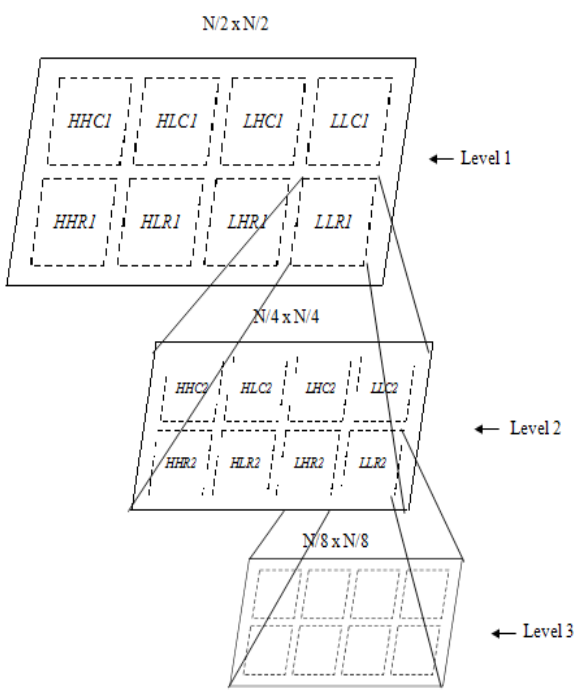

Figure 3. Pyramid structure of DTCWT.
After three levels of decomposition, there are eight sub bands at each level. There are six LH, HL and HH components of real and complex sub bands at each level that capture the six orientations of edges in the input image. The two LL real and complex sub band capture the low frequency components. The decomposed sub bands are in pyramidal form and are organized in hierarchy as shown in Figure 3.

\subsection{Feature Point Extraction}

In this section the process of applying DTCWT for feature point detection is described. Edges in is considered as one of the significant feature in an image. Multi scale edge detectors determine the sharp variations based on first and second derivatives. The extreme points in first derivative correspond to the zero crossing and inflection points in the second derivative. The $2 \mathrm{D}$ dual tree complex wavelet transform of an image $\mathrm{f}(\mathrm{x}, \mathrm{y})$ decomposes the image into two independent directions as in Eq. (2)

$$
\begin{aligned}
& W_{2^{j}}^{1} f(x, y)=f^{*} \varphi_{2^{j}}^{1}(x, y) \\
& \text { and } W_{2^{j}}^{2} f(x, y)=f^{*} \varphi_{2^{j}}^{2}(x, y)
\end{aligned}
$$

At each level the modulus of the gradient is given by Eq. (3),

$$
M_{2 j} f(x, y)=\sqrt{\left|W_{2^{j}}^{1} f(x, y)\right|^{2}+\left|W_{2^{j}}^{2} f(x, y)\right|^{2}}
$$

The gradient vector is threshold and the significant edge points are detected at each scale of $2 \mathrm{j}$ levels. The significant features points obtained for both the images are matched to measure similarity index using the cross correlation function, with rotational effect given by Eq. (4),

$\bar{C}_{f f_{2}}(p ; q ; \theta)=\frac{1}{\sigma_{1} \sigma_{2}(2 M+1)^{2}} \sum_{i, j=-M}^{i, j=+M}\left[f_{1}\left(x+p_{x}, y+p_{y}\right)-\mu_{1}\right]\left[f_{1}\left(x+p_{x}, \hat{y}+p_{y}\right)-\mu_{2}\right]$

The matching window is set to $(2 \mathrm{M}+1)^{2}, \mu$ is the local mean and $\sigma$ is the variance, $\mathrm{x}=\hat{x} \cos \theta-\hat{y} \sin \theta, \mathrm{y}=\hat{x} \sin \theta$ $+\hat{y} \cos \theta$. The orientation of the matched feature points are extracted from $\mathrm{W}$ and $\mathrm{W}$ which is expressed as in Eq. (5),

$$
\operatorname{Arg}\left(W_{2^{j}}^{1} f(x, y)+W_{2^{j}}^{2} f(x, y)\right)
$$

From the features points $\mathrm{FP}_{\mathrm{fl}}=\left\{\mathrm{p}_{\mathrm{i}}=\left(\mathrm{p}_{\mathrm{x}}{ }^{\mathrm{i}}, \mathrm{p}_{\mathrm{y}}\right)^{\mathrm{t}}\right\}_{\mathrm{i}=1,2, \ldots}$ ${ }_{\mathrm{Nf1}}$ and $\mathrm{FP}_{\mathrm{f} 2}=\left\{\mathrm{q}_{\mathrm{i}}=\left(\mathrm{q}_{\mathrm{x}}{ }^{\mathrm{i}}, \mathrm{q}_{\mathrm{y}}\right)^{\mathrm{i}}\right\}_{\mathrm{i}=1,2, \ldots \mathrm{Nf2}}$ the angle of an edge at a given feature point $\mathrm{p}_{\mathrm{i}}$ and $\mathrm{q}_{\mathrm{i}}$ are computed by Eq. (6),

$$
\theta_{i j}=A\left(q_{j}\right)-A\left(p_{i}\right)
$$

The orientation difference between the two image $f_{1}$ and $\mathrm{f}_{2}$ is computed by considering $\theta_{\mathrm{i}, \mathrm{j}}$ and $\mathrm{C}_{\mathrm{fl}, \mathrm{f} 2}$. The distri- 
bution of the number of pairs $\left(\mathrm{p}_{\mathrm{i}} \leftrightarrow \mathrm{q}_{\mathrm{i}}\right)$ at each angle $\theta$ that satisfy the conditions $\theta \mathrm{i}, \mathrm{j}=\theta$ and $\mathrm{C}_{\mathrm{fl}, \mathrm{f2}}\left(\mathrm{p}_{\mathrm{i}} ; \mathrm{q}_{\mathrm{i}}, \theta_{\mathrm{i}, \mathrm{j}) \geq} 0.8\right.$ is computed by the angle histogram $\mathrm{H}(\theta)$. The rotation angle is determined by computing the angle that corresponds to the maximum peak in the histogram. With the orientation difference between the two images being computed, the matching feature point pairs are computed by first rotating all the points in $\mathrm{f}_{1}(\mathrm{x}, \mathrm{y})$ to their new positions in $\hat{f}_{1}(\hat{x}, \hat{y})$, where $\hat{x}=x \cos \bar{\theta}+y \sin \bar{\theta}, \hat{y}=-x \sin \bar{\theta}+y \cos \bar{\theta}$. If the feature point in $\mathrm{f}_{1}$ is rotated to a new position in $\hat{f}$ the angle $\theta$ is also accordingly set to meet the requirements of the coordinates of $\tilde{f}$. For every feature point $\hat{p}$ in $\hat{f}_{1}$ a corresponding matching point $\hat{p}$ forms the matching pair and needs to satisfy two conditions:

Condition 1

$$
\bar{C}_{\hat{f}_{1} \hat{f}_{2}}(\hat{p} ; \tilde{q})=\max _{\tilde{q}_{k} \in F P\left(f_{2}\right)} \max _{\tilde{q}_{n} \in E\left(q_{k}\right)} \bar{C}_{\hat{f}_{1} f_{2}}\left(\hat{p}_{i} ; \tilde{q}_{n}\right)
$$

Condition 2

$$
\bar{C}_{\hat{f}_{1} f_{2}}\left(\hat{p}_{i} ; \tilde{q}\right)>T_{C} \text {, where } T_{C}=0.75
$$

The edge point $\tilde{q} \in \mathrm{E}(\mathrm{qk})$ and $q_{k} \in \mathrm{FPf}_{2}$ is found by maximizing the measure of $\bar{C}_{\hat{f}_{1} \hat{f}_{2}}$ as per condition 1 . Condition 2 enforces the value of $\bar{C}_{\hat{f}_{1} f_{2}}$ of a matching pair to be greater than the set threshold ( 0.6 is set). The search operation is made faster by setting the orientation criterion constraint by setting the orientation difference between $\mathrm{f}_{1}$ and $\mathrm{f}_{2}$ to be less than $10^{\circ}$ (i.e. $\left|\mathrm{A}\left(\mathrm{q}_{\mathrm{j}}\right)-\mathrm{A}\left(\mathrm{p}_{\mathrm{i}}\right)\right|$ $\left.<10^{\circ}\right)$. The orientation criteria constraint improves the processing speed and is suitable for real time image registration. In order to remove the false matching pairs, a modified method is proposed based on $\mathrm{Li}$ et al iterative algorithm. Considering all the matching pairs represented by $M P=\left\{\mathrm{p}_{\mathrm{i}} \leftrightarrow \mathrm{q}_{\mathrm{i}}\right\}_{\mathrm{i}=1,2, \ldots \mathrm{Nm}}\left(\mathrm{p}_{\mathrm{i}}=\left(p_{x}^{i}, p_{y}^{i}\right)\right.$ is a point in $\hat{f}_{1}(\mathrm{x}, \mathrm{y})$ and $\mathrm{q}_{\mathrm{i}}=\left(q_{x}^{i}, q_{y}^{i}\right)$ is a point in $\mathrm{f}_{2}(\mathrm{x}, \mathrm{y})$, (with $\mathrm{N}_{\mathrm{m}}$ representing number of elements in $\mathrm{MP}$ ) the matching pairs are appropriate only if they satisfy the following condition

$$
p_{i}=s q_{i}+T \text { fori }=1,2 \cdots N_{m}
$$

Where, $\mathrm{s}$ and $\mathrm{T}$ are the scalar and translation vectors. The scale $\mathrm{s}$ between $\hat{f}_{1}(\mathrm{x}, \mathrm{y})$ and $\hat{f}_{2}(\mathrm{x}, \mathrm{y})$ is given by $\mathrm{d} 2 / \mathrm{d} 1$ is estimated between two matching pairs in MP. The distance between the two matching pairs is given by Eq. (7),

$$
d 1=\sqrt{\left(p_{x}^{i}-p_{x}^{j}\right)^{2}+\left(p_{y}^{i}-p_{y}^{j}\right)^{2}}
$$

$$
\text { and } d 2=\sqrt{\left(\boldsymbol{q}_{x}^{i}-\boldsymbol{q}_{x}^{j}\right)^{2}+\left(\boldsymbol{q}_{y}^{i}-\boldsymbol{q}_{y}^{j}\right)^{2}}
$$

The translation $\mathrm{T}^{\mathrm{i}}$ between $\mathrm{p}_{\mathrm{i}}$ and $\mathrm{q}_{\mathrm{i}}$ and translation $\mathrm{T}^{\mathrm{j}}$ between $p_{j}$ and $q_{j}$ are computed based on the above equation. If the feature pairs $\left\{\mathrm{p}_{\mathrm{i}} \leftrightarrow \mathrm{q}_{\mathrm{i}}\right\}$ and $\left\{\mathrm{p}_{\mathrm{j}} \leftrightarrow \mathrm{qj}\right\}$ are correct matching pairs the difference between $\mathrm{T}^{\mathrm{i}}$ and $\mathrm{T}^{\mathrm{j}}$ should be small. By setting a threshold, the possible matching pairs that are consistent are selected based on maximum matching similarity or normalized correlation and all other are eliminated. A consistency test is also carried out so that the appropriate pairs are not eliminated. The number of times the matching pair $\left\{\mathrm{p}_{\mathrm{i}} \leftrightarrow \mathrm{q}_{\mathrm{i}}\right\}$ is consistent with other matching pairs is counted and is set to $\mathrm{S}(\mathrm{i})$. A matching pair $\left\{\mathrm{p}_{j} \leftrightarrow \mathrm{q}_{\mathrm{j}}\right\}$ is said to be consistent only if the distance between the translation vectors $\mathrm{T}^{\mathrm{i}}$ and $\mathrm{T}^{\mathrm{j}}$ is less than a threshold. The counter $S$ is incremented by one if the pairs are consistent; the process is carried out until all matching pairs are evaluated. The number of tests required will be $\mathrm{N}_{\mathrm{m}}\left(\mathrm{N}_{\mathrm{m}}-1\right)$. After the consistency test is carried out, each matching pair is associated with a counter value $S$. The matching pair is considered as registration parameter only if the corresponding counter value $\mathrm{S}$ is 2 and greater, all other pairs are discarded. The appropriate set of matching parameters $M=\left\{p_{i} \leftrightarrow q_{i}, i=1,2, \ldots \ldots\right.$ $\mathrm{Nc}$ \} after refinement are used for computation of image transformation parameters such as scaling (s), rotation angle (R) and translation shifts ( $\mathrm{T}$ ) ( $\mathrm{x}$ and y directions) using singular value decomposition (SVD) method, other matching techniques are presented $\mathrm{in}^{\frac{18,19}{2}}$, SVD is most simple and hence is used in this work. The four parameters are related by Eq. (8),

$$
\begin{aligned}
& \boldsymbol{q}_{i}=\mathbf{s R p i}+\mathbf{T} \text { for } \mathbf{i}=1,2,3, \ldots \ldots . \mathbf{N c} \\
& \text { The rotational parameter } R=\left[\begin{array}{cc}
\cos \hat{\theta} & -\sin \hat{\theta} \\
\sin \hat{\theta} & \cos \hat{\theta}
\end{array}\right] \sin \hat{\theta},
\end{aligned}
$$

$\mathrm{T}=\left(\mathrm{t}_{\mathrm{x}}, \mathrm{t}_{\mathrm{y}}\right)^{\mathrm{t}}$ and $\mathrm{P}_{\mathrm{i}}=\left(p_{x}^{\mathrm{i}}, p_{y}^{\mathrm{i}}\right)^{\mathrm{t}}$ is a point in $\hat{f}_{1}(\mathrm{x}, \mathrm{y})$ and $\mathrm{q}_{\mathrm{i}}=\left(q_{x}^{i}, q_{y}^{i}\right) \mathrm{t}$ is a point in $\mathrm{f}_{2}(\mathrm{x}, \mathrm{y}), \theta$ is difference in orientation between $\hat{f}_{1}(\mathrm{x}, \mathrm{y})$ and $\mathrm{f}_{2}(\mathrm{x}, \mathrm{y})$. The transformation parameters are estimated by finding optimal solution by minimizing the expression given by Eq. (9),

$$
\varnothing=\sum_{i=1}^{N_{c}} \boldsymbol{s} \boldsymbol{R} \boldsymbol{p}_{i}+\boldsymbol{T}-\boldsymbol{q}_{i}^{2}
$$

The solution to the above equation is simplified as per the discussions presented in ${ }^{16}$ and is presented as in Eq. (10), 


$$
\begin{gathered}
\varnothing=\sum_{i=1}^{N_{c}} \boldsymbol{q}_{i}^{\sim \boldsymbol{t} \sim} \boldsymbol{q}_{i}^{\sim}-\frac{\left[\sum_{i=1}^{N_{c}} \boldsymbol{q}_{i}^{\sim t} \boldsymbol{R} \boldsymbol{q}_{i}^{\sim}\right]^{2}}{\sum_{i=1}^{N_{c}} \boldsymbol{P}_{i}^{\boldsymbol{t}} \boldsymbol{P}_{i}^{\sim}} \\
\text { Where, }{ }^{\bar{p}=} \frac{\mathbf{1}}{N_{c}} p_{i} \sum_{i=\mathbf{0}}^{N_{c}} p_{i} \text { and } \\
\bar{q}=\frac{\mathbf{1}}{N_{c}} p_{i} \sum_{i=0}^{N_{c}} q_{i} \text { and } S=\frac{\sum_{i=\mathbf{1}}^{N_{c}} q_{i}^{\sim t} R p_{i}}{\sum_{i=\mathbf{1}}^{N_{c}} P_{i}^{t} p_{i}} \text { thus to find }
\end{gathered}
$$

the transformation parameters can be obtained by maximizing the term in Eq. (11),

$$
\varnothing^{\prime}=\left[\sum_{i=1}^{N_{c}} \boldsymbol{q}_{i}^{\sim t} \boldsymbol{R} \boldsymbol{p}_{i}\right]
$$

In order to compute $\mathrm{R}$, as per the SVD method ${ }^{17}$, the 2 $\mathrm{x} 2$ matrix $\mathrm{H}$ is computed as given in Eq. (12),

$$
\boldsymbol{H}=\left[\sum_{i=1}^{N_{c}} \boldsymbol{q}_{i}^{t} \boldsymbol{p}_{i}\right]
$$

The SVD vectors are $\mathrm{H}$ are obtained by computing $\mathrm{H}=\mathrm{P}^{\wedge} \mathrm{Q}^{\mathrm{t}}$ and the rotational parameter is computed by $\mathrm{R}=\mathrm{QP}$. The process of computing transformation parameters is initiated by computing $\mathrm{R}$ and then fine tuning $\theta$. The scaling factor $\mathrm{s}$ and translation vector $\mathrm{T}$ are obtained for image registration. Once the images are registered, there is a need for refinement of registration process. The refinement process is a back annotation process. For each of the $\mathrm{q}_{\mathrm{i}}$ in $\mathrm{f}_{2}$, the corresponding point $\hat{p}_{1}$ in $\hat{f}_{1}$ is identified by searching the pixels in the given window centered at the coordinate pi. A measure of $\mathrm{C}$ given as in Eq. (13),

$$
\boldsymbol{C}_{f_{i, J_{2}}}\left(\boldsymbol{p}_{i}, \boldsymbol{q}_{i}\right)=\max _{\boldsymbol{p} \in N_{i}} \boldsymbol{C}_{f_{\mathrm{i}, J_{2}}}\left(\boldsymbol{p}_{i}, \boldsymbol{q}_{i}\right) \boldsymbol{C}_{f_{\mathrm{i}, J_{2}}}\left(\boldsymbol{p}_{i}, \boldsymbol{q}_{i}\right) \geq 0.75
$$

$\mathrm{C}$ determines the closeness of these points. If the difference is large than the set threshold (0.75), then the feature points are re-sampled for computation. The refinement process is carried out only with few sets of samples, with $s^{\prime}, \theta^{\prime}, t^{\prime}{ }_{x}$ and $t^{\prime}{ }_{y}$ more accurate values of $\mathrm{s}, \theta, \mathrm{tx}$, ty are arrived at. The fine-tuned feature set is given by for the given feature set

$$
\begin{aligned}
& \left(s, \theta, t_{x_{x}}, t_{y}\right)^{t}=\left(s s^{\prime}, \theta+\theta^{\prime}, s^{\prime} \cos \theta^{\prime} t_{x}+s^{\prime} \sin \theta^{\prime} t_{y}+t^{\prime} x-s^{\prime} \sin \theta^{\prime} t_{x}+s^{\prime} \cos \theta^{\prime} t_{y}+t^{\prime} y\right)^{\prime} \\
& q_{i}=s^{\prime} R\left(\theta^{\prime}\right) p_{i}+T \\
& q_{i}=s^{\prime} R\left(\theta^{\prime}\right)\left[s R(\theta) p_{i}+T\right]+T^{\prime} \\
& q_{i}=s^{\prime} R\left(\theta+\theta^{\prime}\right) p_{i}+\left[s^{\prime} R\left(\theta^{\prime}\right) T+T^{\prime}\right]
\end{aligned}
$$

The registration process is iterative and is continuously processed until the results are satisfactory.

\section{Results and Discussion}

The proposed algorithm based in DTCWT is modeled in MATLAB and the images supplied in MATLAB have been used for validation of proposed algorithm.

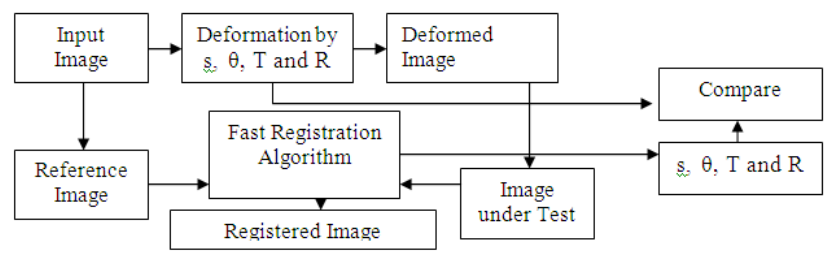

Figure 4. Experimental setup for performance evaluation.
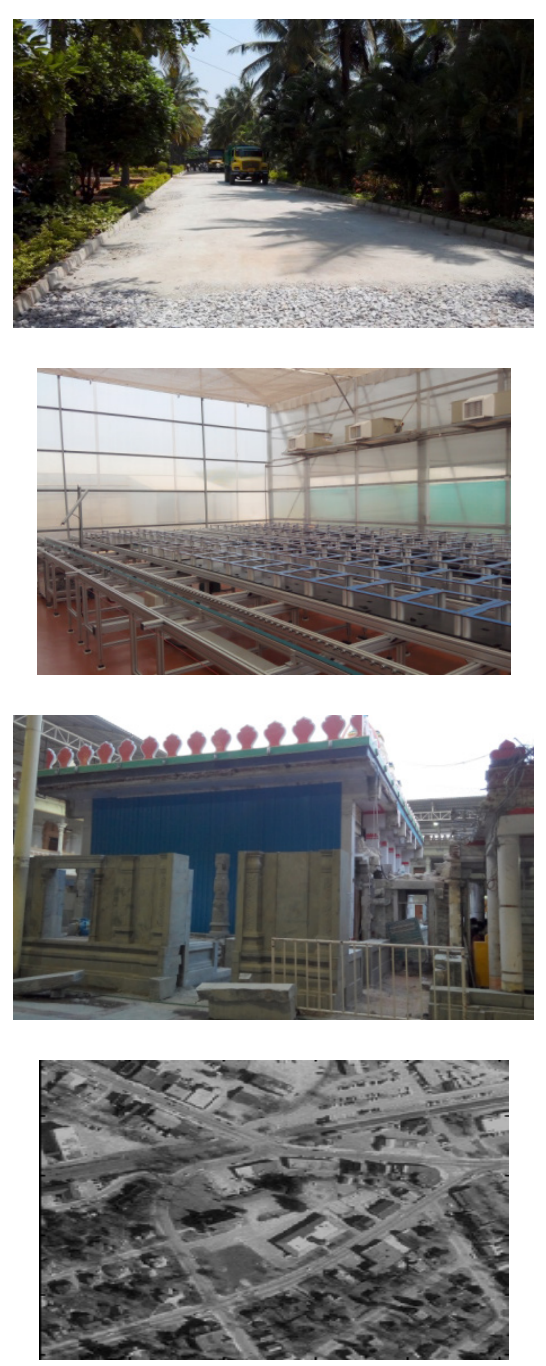

Figure 5. Test Images (a) Road, (b) Plant (c) Mantralaya (d) Ordorthophoto.

Two images are considered, the reference image 'westconcordorthophoto, is a georegistered panchromatic 
gray scale image supplied by Massachusetts Geographic Information System (MassGIS). The input image is 'westconcordaerial' which is aerial photographic image supplied by $m$ Power3/Emerge. The experimental setup for image registration is shown in Figure 4. The input image is considered as reference image, the input image is deformed by scaling, translation and rotation with known set of parameters. The input image after scaling is considered as image under test which needs to be registered with the reference image. The registration process is carried out based on the proposed technique discussed in the previous section. The transformation parameters s, $\theta, \mathrm{T}$ and $\mathrm{R}$ are computed and are compared with the parameters $\mathrm{s}, \theta, \mathrm{T}$ and $\mathrm{R}$ that were used to deform the reference image. The similarity index of comparison is used as performance measure for the proposed method. Figure 5 shows the image data sets considered for image registration. The color images are converted to gray scale and are smoothened by using histogram technique. The enhanced images are considered as reference images.

The test image ordorthophoto is registered with the translated, rotated and scaled image which is shown in Figure 6 . The points A, B and C represented in the Figure 6 indicate the deformation in the input image with regard to the reference image. The deformations are introduced are recorded and tabulated in the Table 1.

Table 1. Transformation Parameters

\begin{tabular}{|c|c|c|c|c|c|}
\hline Images & Transformation & Scale: $\mathrm{s}$ & Translation: $t x$ & Translation: ty & Rotation: $\theta^{0}$ \\
\hline \multirow[t]{2}{*}{ Ordorthophoto } & Before registration & 0.9123 & 82 & 156 & 21.29 \\
\hline & After registration & 0.9127 & 78 & 154 & 21.42 \\
\hline \multirow[t]{2}{*}{ Road } & Before registration & 1.1 & 180 & 396 & 8.61 \\
\hline & After registration & 0.97 & 178 & 380 & 85 \\
\hline \multirow[t]{2}{*}{ Plant } & Before registration & 1.21 & 510 & 364 & 34.3 \\
\hline & After registration & 1.2 & 500 & 366 & 33.8 \\
\hline \multirow[t]{2}{*}{ Mantralaya } & Before registration & 0.8 & 408 & 126 & 47.1 \\
\hline & After registration & 0.712 & 411 & 121 & 47.8 \\
\hline
\end{tabular}

Table 2. Comparison of transformation parameters

\begin{tabular}{|c|c|c|c|c|c|c|c|c|c|c|c|c|}
\hline \multirow[t]{2}{*}{ Image } & \multicolumn{3}{|c|}{ Input deformations } & \multicolumn{3}{|c|}{ Christopher ${ }^{15}$} & \multicolumn{3}{|c|}{ Le MoigneJ. ${ }^{1}$} & \multicolumn{3}{|c|}{ This work } \\
\hline & tx & ty & $\theta^{0}$ & $t x$ & ty & $\theta^{0}$ & tx & ty & $\theta^{0}$ & tx & ty & $\theta^{0}$ \\
\hline Road 1 & 50 & 50 & -30 & 40 & 40 & -20 & 45 & 55 & -28 & 48 & 52 & -29 \\
\hline Road 2 & 25 & 25 & -15 & 15 & 15 & -05 & 22 & 21 & -15 & 24 & 24 & -15 \\
\hline Road 3 & -50 & -50 & -15 & -38 & -40 & -11 & -42 & -56 & -12 & -45 & -54 & -14 \\
\hline Road 4 & -25 & -25 & -30 & -18 & -35 & -35 & -29 & -24 & -36 & -27 & -25 & -32 \\
\hline Plant 1 & 10 & 20 & 20 & 16 & 26 & 25 & 14 & 23 & 26 & 12 & 22 & 24 \\
\hline Plant 2 & 20 & 10 & 10 & 17 & 20 & 20 & 15 & 15 & 14 & 17 & 11 & 12 \\
\hline Plant 3 & -5 & -10 & -20 & -9 & -19 & -09 & -7 & -14 & -24 & -6 & -11 & -21 \\
\hline Plant 4 & -10 & -5 & -10 & -16 & -5 & -7 & -12 & -4 & -6 & -9 & -3 & -7 \\
\hline Mantralaya 1 & 12 & 7 & 7 & 17 & 15 & 7 & 15 & 5 & 10 & 15 & 5 & 9 \\
\hline Mantralaya 2 & 22 & 12 & 10 & 10 & 8 & 16 & 27 & 15 & 10 & 25 & 11 & 10 \\
\hline Mantralaya 3 & -32 & -14 & -12 & -38 & -21 & -15 & -31 & -15 & -14 & -31 & -15 & -11 \\
\hline Mantralaya 4 & -42 & -22 & -26 & -38 & -15 & -20 & -41 & -18 & -22 & -42 & -21 & -23 \\
\hline Ordorthophoto1 & 7 & 15 & 11 & 7 & 14 & 21 & 7 & 12 & 13 & 7 & 11 & 13 \\
\hline Ordorthophoto 2 & 15 & 26 & 1 & 04 & 16 & 1 & 12 & 22 & 2 & 16 & 24 & 2 \\
\hline Ordorthophoto3 & -13 & -33 & -2 & -6 & -25 & -2 & -17 & -38 & -2 & -15 & -36 & -2 \\
\hline Ordorthophoto4 & -23 & -42 & -6 & -17 & -32 & -6 & -26 & -47 & -8 & -21 & -44 & -8 \\
\hline
\end{tabular}




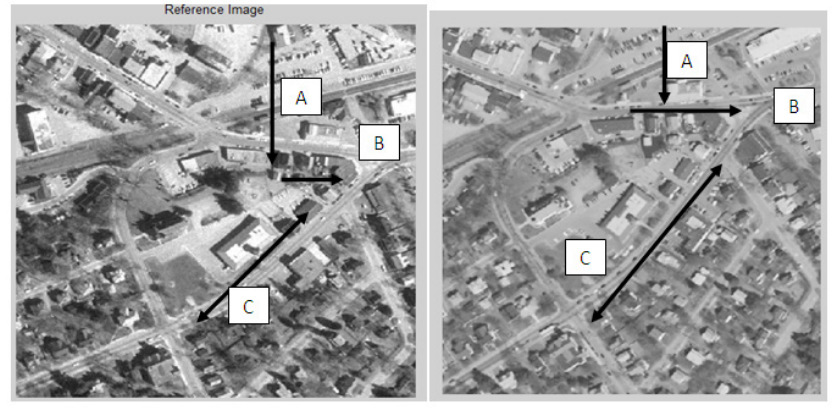

Figure 6. Input image under test (a) Reference image (b) Deformed image.

Table 2 compares the performances of various registration algorithms, the proposed work demonstrates the accuracy in estimation transformational parameters.

Figure 7 presents the results of registered image that is superimposed with the reference image. The images presented with the deformations of $\mathrm{A}, \mathrm{B}$ and $\mathrm{C}$ is minimized in the registered image.

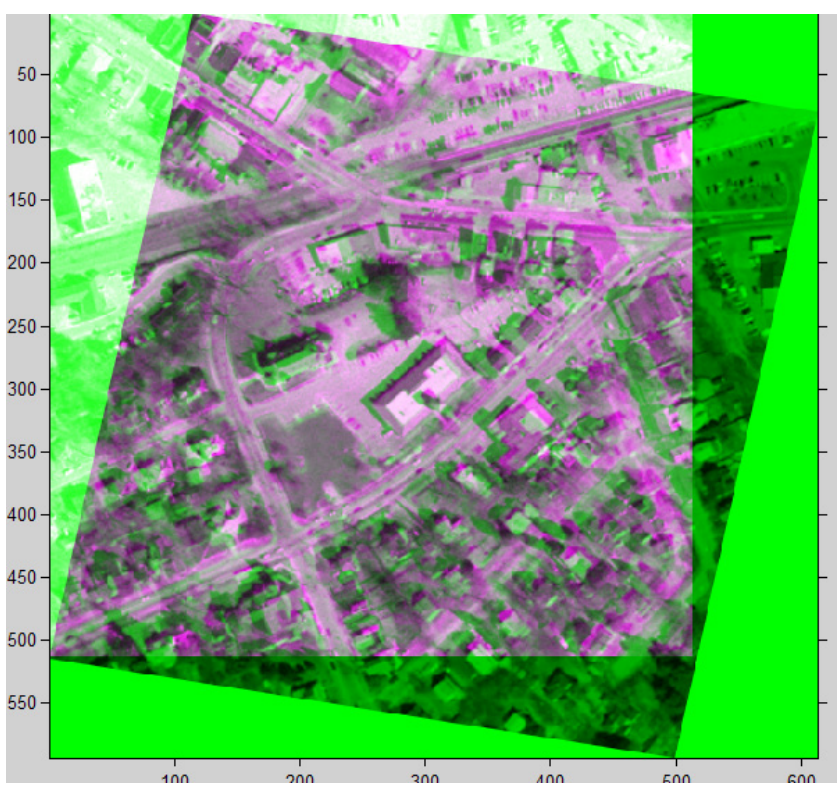

Figure 7. Registered images.

The proposed method uses DTCWT transformation for extraction of features; the six sub band components are suitable for extraction of features from three orientations. The features are extracted from the lowest real sub band; the lowest imaginary sub band can also be used for registration. The real high frequency sub bands are used to extract the features after thresholding, however they are feature pairs could not lead to significant improvement in registration performance. It needs to be explored to sue multi-level sub bands for significant feature extraction. The total time estimated for registration is found to be of 30 units. With robust feature samples, the time was estimated to be around 128 time units. Thus with the proposed method the feature sets were capable of performing registration with minimum of $32 \mathrm{~dB}$ PSNR with optimum feature pairs identified.

\section{Conclusion}

In this work, a novel technique for image registration is presented based on dual tree complex wavelet theory. The input image is pre-processed with Gaussian filters to remove noise; the filtered image is transformed using wavelet filter to compute multiple sub bands. From the multiple sub bands, Gradient is computed using Sobel operator; from the features obtained, the reference points that are obtained after averaging provide the accurate points for image transformation and registration. The proposed algorithm is verified for its performances with reference satellite image and the results obtained demonstrate the novelty in the algorithm proposed. For real time application it is required to improve the performances of computation speed of DTCWT and IDTCWT, Gaussian filtering and gradient operation.

\section{References}

1. Moigne JL, Campbell W, Cromp R. An automated parallel image registration technique based on the correlation of wavelet features. IEEE Transactions on Geoscience and Remote Sensing. 2002 Aug; 40(8):1849-64.

2. Fonseca L, Costa M. Automatic registration of satellite images. Proceedings of the Brazilian Symposium on Computer Graphic and Image Processing, Brazil. 1997 Oct 14-17; 219-26.

3. Zheng Q, Chellappa R. A computational vision approach to image registration. IEEE Transactions on Image Processing. 1993 Jul; 2(3):311-26.

4. Li H, Zhou Y. Automatic EO/IR sensor image registration. IEEE Int Conf on Image Proc.1995; 2. p. 161-64.

5. Quddus, Basir O. Wavelet-Based Medical Image Registration for Retrieval Applications. Proceedings of the 2008 International Conference on BioMedical Engineering and Informatics. IEEE Computer Society. 2008 Feb. p. 301-05.

6. Wong A, Clausi D. Automatic Registration of Inter-band and Inter-sensor Images using Robust Complex Wavelet Feature Representations. Proc of 5th IAPR Workshop on Pattern Recognition in Remote Sensing. 2008. 
7. Kingsbury NG. The dual-tree complex wavelet transform: a new efficient tool for OFDM restoration and enhancement. Proc EUSIPCO Rhodes.1998; 319-22.

8. Kingsbury NG. Complex wavelets for shift invariant analysis and filtering of signals. Applied and computational harmonic analysis. 2001; 234-53.

9. Kingsbury NG. Design of q-shift complex wavelets for image processing using frequency domain energy minimization. Proc IEEE Int Conf Image Processing, Barcelona. 2003; 1. p. 1013-16.

10. Kingsbury NG. Image processing with complex wavelets, (Silverman B, Vassilicos J editors, Wavelets): The key to intermittent Information, Oxford University Press, USA. 1999; 165-85.

11. Romberg J, Choi H, Baraniuk R, Kingsbury NG. Multiscale classification using Complex wavelets, Proc. ICIP, Vancouver. 2000.

12. Kingsbury NG. A dual-tree complex wavelet transform with improved orthogonality and symmetry properties. Proc IEEE Int Conf Image Processing, Vancouver, Canada. 2000 Sep 10-13; 2:375-8.

13. Kingsbury NG. The dual-tree complex wavelet transform: a new technique for shift invariance and directional filters. IEEE Digital Signal Processing Workshop. 1998; 86.
14. Ghantous M, Ghosh SBayoumi A. A multi-modal automatic image registration technique based on complex wavelets. Journal of Signal Processing Systems. 2003; 71(1):41-55.

15. Paulson C, Ezekiel S, Wu D. Wavelet-based image registration. Proc SPIE 7704, Evolutionary and Bio-Inspired Computation: Theory and Applications. 2010.

16. Umeyama S. Least-squares estimation of transformation parameters between two point patterns. IEEE Trans Pattern Anal Machine Intel. 1991; PAMI-13:376-80.

17. Arun KS, Huang TS, Blostein SD. Least-square fitting of two 3-D point sets. IEEE Trans Pattern Anal Mach Intel. 1987; PAMI-9(5):698-700.

18. Pavani T, Das RP, Jyothi AN, Murthy ASD. Investigations on Array Pattern Synthesis using Nature Inspired Metaheuristic Algorithms. Indian Journal of Science and Technology. 2016 Jan; 9(2):1-11.

19. Manju R, Nargunam AS. Estimation of Performance in Multimodal Biometric based Authentication System using Various Clustering. Indian Journal of Science and Technology. 2016 Mar; 9(13):1-7. 